\title{
AN EXACT SOLUTION OF THE DIRAC OSCILLATOR PROBLEM IN THE CONTEXT OF GENERALIZED UNCERTAINTY PRINCIPLE
}

\author{
Md. Arifuzzaman ${ }^{1}$, Md. Moniruzzaman', S. B. Faruque ${ }^{3}$ \\ ${ }^{1}$ Lecturer, Department of Electrical and Electronic Engineering, International Islamic University Chittagong, \\ Chittagong-4203, Bangladesh, arifphy_uzzaman@yahoo.com \\ ${ }^{2}$ Assistant Professor, Department of Physics, Mawlana Bhashani Science and Technology University, Santosh, \\ Tangail-1902, Bangladesh, monir_m17@yahoo.com \\ ${ }^{3}$ Professor, Department of Physics, Shah Jalal University of Science and Technology, Sylhet-3114, Bangladesh, \\ awsbf62@yahoo.com
}

\begin{abstract}
In this paper, we present an exact solution of the Dirac oscillator problem in one dimension in the context of the generalized uncertainty principle (GUP). The solution method presented here depends on the knowledge of the energy eigenvalues of the quantum harmonic oscillator with GUP. The crucial property of harmonic oscillator that the kinetic energy and the potential energy part of the Hamiltonian are of equal weight is used to obtain exact energy spectrum. Our result coincides with the results found in the literature. However, the solution procedure is completely different from others, very handy and alternative one. Moreover, we also remark the super symmetry aspects of the system.
\end{abstract}

Keywords: Dirac Oscillator; Generalized Uncertainty Principle; a Minimal Length.

$* * *$

\section{INTRODUCTION}

In the view point of quantum mechanics, Kempf et al [1-4] has modified the usual canonical commutation relation between position and momentum operators as $[x, p]=i \hbar\left(1+\beta p^{2}\right)$, where $\beta$ is a small positive parameter called the deformation parameter so that a minimal length can be introduced as an additional uncertainty in position measurement. A non-zero minimal position uncertainty has appeared in the context of the string theory [5-10] which arises due to the fact that the string cannot have probe distances smaller than the string scale $\hbar \sqrt{\beta}$. Non-commutative geometries [11], black hole physics [12] and the holographic principle [13] also furnish the more strong evidence for the occurrence of the minimal length. This modified commutation relation leads to the standard Heisenberg uncertainty relation as $\Delta x \Delta p \geq \frac{\hbar}{2}\left[1+\beta(\Delta p)^{2}\right]$, which evidently implies the existence of a non-zero minimal length, $\Delta x \geq \hbar \sqrt{\beta}$.This modification of uncertainty relation is usually termed the generalized uncertainty principle (GUP) [14] or the minimal length uncertainty principle. In the context of the GUP the momentum operator acts as

$$
p_{o p}=-i \hbar \frac{\partial}{\partial x}\left[1+\frac{\beta}{3}\left(-i \hbar \frac{\partial}{\partial x}\right)^{2}\right] \text { and the position }
$$

operator remains the usual operator $x_{o p}=x$ for configuration space [2].

At the present time, one of the common aspects of the notion of the GUP is to unify quantum mechanics with general relativity in the realm of quantum gravity [15]. Another is the minimal length may regularize unwanted divergences in the quantum field theory [16]. The minimal length also may be of great concern in non-relativistic quantum mechanics. In reality, it has been conjectured that the minimal length can be inferred as an intrinsic scale characterizing the structure of the considered system and its finite size under study [16]. Accordingly, the formalism of the GUP can also propose a most up-to-date model for effective description of complex systems such as quasi-particles and various collective excitations in solid or composite particles, such as molecules, nuclei, and nucleons $[4,17]$.

Many authors at present engage to study the influence of GUP on quantum mechanical problems and a large number of problems have been studied in association with the GUP over the last decade: The harmonic oscillator problem in arbitrary dimensions has been solved exactly in [18]. An approximate energy spectrum of the hydrogen atom in presence of the 
minimal length has been obtained in coordinate space in [19]. The implications of a generalized uncertainty relation on cosmology have been discussed in many papers; for example, see [20-22]. The roles of GUP on generalization of the electromagnetic field have been studied in [23]. The dynamics of a particle in a gravitational quantum well in the framework of non-relativistic quantum mechanics with GUP has been discussed in [24]. The free particle Dirac equation with GUP has been solved in [25]. Dirac oscillator in three dimensions has been analyzed in [26]. Some aspects of the onedimensional harmonic oscillator in GUP picture have been studied in [27, 28]. Quantum mechanical scattering problem for the Yukawa and the Coulomb potential has been studied in [29]. Under the influence of the GUP, the dominant contribution to the energy levels of the $(1+1)$ dimensional Dirac equation with a linear scalar-like potential has been obtained approximately in [30]. The energy spectrums of the $(1+1)$ dimensional Dirac equation with mixed vector-scalar linear potentials in [31] and a linear scalar-like potential in [32] have been obtained exactly. The exact energy spectrum of a relativistic electron in the presence of a static magmatic field and in the scenario of GUP has been obtained in [33].

In this paper, we present an exact solution of the Dirac oscillator problem in one dimension in the context of the GUP and along with the context of the usual Heisenberg uncertainty principle. We show an easy and simpler method to find the energy spectrum. The method depends on the knowledge of the energy spectrum of harmonic oscillator with GUP. We also implement the property of harmonic oscillator, the kinetic energy and the potential energy part of the Hamiltonian are of equal weight, to obtain exact energy spectrum. Our result fully coincides with existing result in the literature [34]. Therefore, this paper presents a handy alternative method of an exact solution of the Dirac oscillator in one dimension with GUP. The paper also manifests the super symmetry aspects of the system. The Dirac oscillator is important since it possesses physical applications in semiconductor physics [35].

The paper is arranged as follows: In section 2, at first we simplify the Dirac oscillator problem in one dimension, where the problem is reduced to two coupled differential equations. Then we solve the coupled equations in the framework of the usual Heisenberg uncertainty principle in sub section 2.1 and the GUP in sub section 2.2 and we obtain the exact energy spectrum and the super symmetry aspects of the system. Finally in section 3 , we give a brief conclusion.

\section{EXACT SOLUTION OF THE DIRAC OSCILLATOR IN ONE-DIMENSION}

The stationary equation describing the Dirac oscillator in one dimension is given by [36]

$$
c \alpha(p-i \beta m \omega x) \psi+\beta m c^{2} \psi=E \psi
$$

where $\mathrm{m}$ is the rest mass, $\omega$ is the classical frequency of the oscillator and $\psi$ is a two-component spinor. Note that equation (1) is obtained from the Dirac equation in one dimension by the substitution $p \rightarrow p-i \beta m \omega x$.

We use following representation of the Dirac matrices:

$$
\alpha=\sigma_{y}, \beta=\sigma_{z}
$$

and use tow component form of $\psi$ as

$$
\psi=\left(\begin{array}{l}
\varphi_{1} \\
\varphi_{2}
\end{array}\right)
$$

We obtain the following two coupled equations for $\varphi_{1}$ and $\varphi_{2}$ :

$$
\left\{p^{2}+m^{2} \omega^{2} x^{2}+i m \omega[x, p]\right\} \varphi_{1}=\left(\frac{E^{2}}{c^{2}}-m^{2} c^{2}\right) \varphi_{1}
$$

and

$$
\left\{p^{2}+m^{2} \omega^{2} x^{2}-i m \omega[x, p]\right\} \varphi_{2}=\left(\frac{E^{2}}{c^{2}}-m^{2} c^{2}\right) \varphi_{2}
$$

\subsection{IMPLICATIPON OF THE USUAL HEISENBERG UNCERTAINTY PRINCIPLE}

Now, we attend to the problem of solving equations (4.1) and (4.2) in the context of the usual Heisenberg algebra. Using $[x, p]=i \hbar$ in equations (4.1) and (4.2) we obtain the fallowing simultaneous equations

$$
\left\{\frac{p^{2}}{2 m}+\frac{1}{2} m \omega^{2} x^{2}\right\} \varphi_{1}=\left\{\frac{E^{2}}{2 m c^{2}}-\frac{m c^{2}}{2}+\frac{\hbar \omega}{2}\right\} \varphi_{1}
$$

and

$$
\left\{\frac{p^{2}}{2 m}+\frac{1}{2} m \omega^{2} x^{2}\right\} \varphi_{2}=\left\{\frac{E^{2}}{2 m c^{2}}-\frac{m c^{2}}{2}-\frac{\hbar \omega}{2}\right\} \varphi_{2}
$$

Both equations of (5.1) and (5.2) are the equation of harmonic oscillators with frequency $\omega$. Hence, we find from equation

(5.1) and (5.2) for the spectrum of $\varphi_{1}$, the following:

$$
E_{n}= \pm \sqrt{2 n m \hbar \omega c^{2}+m^{2} c^{4}} \quad, \quad n=0,1,2,3, . .
$$


and for the spectrum of $\varphi_{2}$, the following:

$$
E_{n^{\prime}}= \pm \sqrt{2\left(n^{\prime}+1\right) m \hbar \omega c^{2}+m^{2} c^{4}} \quad n^{\prime}=0,1,2,3, . .
$$

The spectra associated with $\varphi_{2 n^{\prime}} \quad$ [equation (6.2)] is exactly the same as that of $\varphi_{1 n}$ [equation (6.1)] except that $\varphi_{1 n}$ possesses an extra level, the base state $(n=0)$. Hence, $\varphi_{1 n}$ and $\varphi_{2 n^{\prime}}$ are super symmetric partners, though very simple, where $\varphi_{1 n}$ and $\varphi_{2 n^{\prime}}$ are the wave function of simple harmonic oscillator.

\subsection{IMPLICATION OF THE GENERALIZED UNCERTAINTY PRINCIPLE}

Now, we attend to the problem of solving equations (4.1) and (4.2) with the generalized Heisenberg algebra. Upon substitution of the generalized Heisenberg algebra $[x, p]=i \hbar\left(1+\beta p^{2}\right)$, in equations (4.1) and (4.2), these equations reduce to the following forms respectively:

$$
\left\{(1-\beta m \hbar \omega) \frac{p^{2}}{2 m}+\frac{1}{2} m \omega^{2} x^{2}\right\} \varphi_{1}=\left\{\frac{E^{2}}{2 m c^{2}}-\frac{m c^{2}}{2}+\frac{\hbar \omega}{2}\right\} \varphi_{1}
$$

and

$$
\left\{(1+\beta m \hbar \omega) \frac{p^{2}}{2 m}+\frac{1}{2} m \omega^{2} x^{2}\right\} \varphi_{2}=\left\{\frac{E^{2}}{2 m c^{2}}-\frac{m c^{2}}{2}-\frac{\hbar \omega}{2}\right\} \varphi_{2}
$$

Remark that the factors $1 \mp \beta m \hbar \omega$ which occurs as multiplications of kinetic energy operator are unit less quantities and except the factors both equations (7.1) and (7.2) are deformed harmonic oscillator i.e. harmonic oscillator with GUP. Thus, we can use the property of harmonic oscillator to obtain the energy levels. For harmonic oscillator, the kinetic energy part of the Hamiltonian is of equal weight to the potential energy part, to obtain the energy levels. Classically, on average the potential energy is equal to the kinetic energy. To obtain the spectra, we can employ this feature in the following way [32]: Divide the spectra of deformed harmonic oscillator in two equal parts and multiply one of the two equal parts by the factor $(1-\beta m \hbar \omega)$ for equation (7.1) and $(1+\beta m \hbar \omega)$ for equation (7.2). Now, the energy levels of deformed harmonic oscillator are [18]

$$
\begin{aligned}
& E_{n}=\left(n+\frac{1}{2}\right) \hbar \omega+\left(n^{2}+n+\frac{1}{2}\right) \frac{\beta m \hbar^{2} \omega^{2}}{2}, \\
& n=0,1,2,3, . .
\end{aligned}
$$

Hence, the preconception as mentioned and neglecting the term of highest order $\beta$, we have for equation (7.1)

$$
\begin{aligned}
& E_{n}= \pm m c^{2} \sqrt{1+2 n \frac{\hbar \omega}{m c^{2}}+n^{2} \frac{\beta \hbar^{2} \omega^{2}}{c^{2}}}, \\
& n=0,1,2,3, . .
\end{aligned}
$$

And for equation (7.2)

$$
\begin{aligned}
& E_{n^{\prime}}= \pm m c^{2} \sqrt{1+2\left(n^{\prime}+1\right) \frac{\hbar \omega}{m c^{2}}+\left(n^{\prime}+1\right)^{2} \frac{\beta \hbar^{2} \omega^{2}}{c^{2}}}, \\
& n^{\prime}=0,1,2,3, . .
\end{aligned}
$$

The spectrum of the Dirac oscillator in presence of the minimal length is given by both equations (9.1) and (9.2). The energy spectrum given by equation (9.1) fully coincides the spectrum obtained in [15]. Note that equation (9.1) gives an extra state at $n=0$, which is an upshot of the existence of the minimal length. This is a manifestation of super symmetry. If we set the deformation parameter $\beta=0$ in equations (9.1) and (9.2) then the equations coincide with the equations $(6.1)$ and (6.2) respectively.

\section{CONCLUSIONS}

In this paper, the Dirac oscillator problem has been reduced to two coupled differential equations. We have presented a comprehensive procedure to solve the coupled equations in the framework of the usual Heisenberg uncertainty principle. Under the framework of the GUP, the coupled equations can be cast into the form of deformed harmonic oscillator i.e. the form of harmonic oscillator with GUP with additionally a unit less factor take place as a multiplication of kinetic energy operator. We have the crucial property of harmonic oscillator that is the kinetic energy and the potential energy part of the Hamiltonian are of equal weight. Using the energy spectrum of deformed harmonic oscillator i.e. harmonic oscillator with GUP, we have obtained the energy spectrum of the coupled equations. The expression of the energy spectrum contains n2dependence term as the energy levels of a particle confined in a potential well which is a consequence of the presence of minimal length. Our obtained energy spectrums are supported 
with the existing energy spectrum found in [34]. Additionally, we obtain an extra energy level with quantum number $n=0$ which is another consequence of the presence of minimal length. This reveals a manifestation of super symmetry of the system.

In conclusion, we present a simpler alternative method of exact solution of the Dirac oscillator in one dimension in the perspective of the GUP. The solution procedure becomes simpler due to apply of the property of simple harmonic oscillator, the kinetic energy and the potential energy part of the Hamiltonian are of equal weight, to obtain exact energy spectrum. Finally, we conclude that we have presented a piece of information to the scientific society.

\section{REFERENCES:}

[1]. A. Kempf, J. Math. Phys. 35, 4483 (1994).

[2]. A. Kempf, G. Mangano, R.B. Mann, Phys. Rev. D 52, 1108 (1995).

[3]. H. Hinrichsen, A. Kempf, J. Math. Phys. 37, 2121 (1996).

[4]. A. Kempf, J. Phys. A 30, 2093 (1997).

[5]. D. Amati, M. Ciafaloni and G. Veneziano, Phys. Lett. B 197, 81 (1987).

[6]. D. J. Gross and P. F. Mende, Phys. Lett. B. 197129 (1987).

[7]. D. J. Gross and P. F. Mende, Nucl. Phys. B 303, 407(1988).

[8]. D. Amati, M. Ciafaloni and G. Veneziano, Int. J. Mod. Phys. A 7, 1615 (1988).

[9]. D. Amati, M. Ciafaloni, and G. Veneziano, Phys. Lett. B.216, 41 (1989).

[10]. E. Witten, Phys. Today 49, 24(1997).

[11]. R. J. Szabo, Phys. Rep. 378, 207 (2003).

[12]. T. Padmanabhan, T.R. Seshadri, T.P. Singh, Int. J. Mod. Phys. A, 1491 (1986).

[13]. L. Susskind, J. Math. Phys. 36, 6377 (1995).

[14]. J. Y. Bang and M. S. Berger, Phys. Rev. D 74, 125012 (2006).

[15]. L. J. Garay, Int. J. Mod. Phys. A 10, 145 (1995).

[16]. D. Bouaziz, M. Bawin, phys. Rev A 76, 032112 (2007).

[17]. R. Sastry Ramchander, J. Phys. A 33, 8305 (2000).

[18]. L. N. Chang, D. Minic, N. Okamura and T. Takeuchi, Phys. Rev. D 65, 125027(2002).

[19]. M. M Stesko, Phys. Rev. A 74, 062105 (2006).

[20]. M. V. Battisti and G. Montani, Phys. Rev. D 77, 023518 (2008).

[21]. M. V. Battisti and G. Montani, Phys. Lett. B 656, 96 (2007)

[22]. K. Nozari, B. Fazlpour, Gen. Rel. Grav. 38, 1661 (2006).

[23]. A. Camacho, Gen. Rel. Grav. 35, 1153 (2003).

[24]. F. Brau and F. Buisseret, Phys. Rev. D 74, 036002 (2006).

[25]. K. Nozari, Chaos Solitons Fractals 32, 303 (2007).

[26]. C. Quesne, V. M. Tkachuk J. Phys. A 38, 1747 (2005).

[27]. Kh. Nouicer, Phys. Lett. A 354, 399 (2006).
[28]. K. Nozari, T. Azizi, Gen. Rel. Grav. 38, 325 (2006). [29]. M. M. Stesko, V. M. Tkachuk, Phys. Rev. A 76, 012707 (2007).

[30]. Md. Sakhawat Hossain, S.B. Faruque, Phys. Scr.78, 035006 (2008).

[31]. Y. Charghi, A. Trabelsi, L. Chetouani, Phys, Lett. A 374, 531-534 (2010).

[32]. Momotaj Ara, Md. Moniruzzaman and S. B. Faruque, Phys. Scr. 82, 035005 (2010).

[33]. Md. Moniruzzaman and S. B. Faruque, Phys. Scr. 85, 035006 (2012), Phys. Scr. 86, 039503 (2012).

[34]. Kh. Noucier, J. Phys. A 39, 5125 (2006).

[35]. R. Renan, M. H. Pacheco and C. A. Almeida, J. Phys.A: Math. Gen. 33, L590 (2000).

[36]. M. Moshinsky and Y. F. Simon, Contemporary Concepts in Physics Series, 9th Vol. (Harwood Academic, New York, 1996).

\section{BIOGRAPHIES}

Md. Arifuzzaman obtained his Bachelor's and Master's degree in Physics from the faculty of Physical Sciences, Shah Jalal University of Science and Technology, Bangladesh in 2009. After completing his graduation, He has been working as a faculty member in Physics under the department of Electrical and Electronic Engineering at International Islamic University Chittagong, Bangladesh since 2009.

Md. Moniruzzaman obtained his Bachelor's and Master's degree in Physics from the faculty of Physical Sciences, Shah Jalal University of Science and Technology, Bangladesh in 2010. At present, $\mathrm{He}$ is working as an Assistant Professor in Physics under the faculty of Science at Mawlana Bhashani Science and Technology University, Santosh, Tangail-1902, Bangladesh.

Dr. Syed Badiuzzaman Faruque is the Professor in the Department of Physics under the faculty of Physical Sciences at Shah Jalal University of Science and Technology, Bangladesh. His area of research interest in the fields of Gravitational Physics and Quantum Mechanics. He has published about 25 research articles in various internationally reputed journals. 